Check for updates

${ }^{1}$ Laboratory of Physiological

Sciences and Department of

Pharmacy of the Affiliated

Hospital of Guangdong Medical

University, Guangdong Medical

University, Zhanjiang, China

${ }^{2}$ Department of Pharmacy, Sun

Yat-Sen Memorial Hospital, Sun

Yat-Sen University, Guangzhou,

China

${ }^{3}$ Department of Pharmacology, Guangdong Medical University,

Zhanjiang, China

${ }^{4}$ Department of Respiratory

Medicine, Affiliated Hospital of

Guangdong Medical University,

Guangdong Medical University,

No 57 , South of Renmin

Avenue, Zhanjiang 524001

China

Correspondence to: W Yao yaoweimin2014@yeah.net

Additional material is published online only. To view please visit the journal online.

Cite this as: BMJ 2018;363:k4388 http://dx.doi.org/10.1136/bmj.k4388

Accepted: 5 October 2018

\section{Triple therapy in the management of chronic obstructive pulmonary disease: systematic review and meta-analysis}

\author{
Yayuan Zheng, ${ }^{1}$ Jianhong Zhu, ${ }^{2}$ Yuyu Liu, ${ }^{3}$ Weiguang Lai, ${ }^{4}$ Chunyu Lin, ${ }^{4}$ Kaifen Qiu, ${ }^{2}$ Junyan Wu, \\ Weimin $\mathrm{YaO}^{4}$
}

\section{ABSTRACT}

OBJECTIVE

To compare the rate of moderate to severe exacerbations between triple therapy and dual therapy or monotherapy in patients with chronic obstructive pulmonary disease (COPD).

DESIGN controlled trials.

\section{DATA SOURCES}

PubMed, Embase, Cochrane databases, and clinical trial registries searched from inception to April 2018.

\section{ELIGIBILITY CRITERIA}

Randomised controlled trials comparing triple therapy with dual therapy or monotherapy in patients with COPD were eligible. Efficacy and safety outcomes of interest were also available.

\section{DATA EXTRACTION AND SYNTHESIS}

Data were collected independently. Meta-analyses were conducted to calculate rate ratios, hazard ratios, risk ratios, and mean differences with $95 \%$ confidence intervals. Quality of evidence was summarised in accordance with GRADE methodology (grading of recommendations assessment, development, and evaluation).

RESULTS

21 trials (19 publications) were included. Triple therapy consisted of a long acting muscarinic antagonist (LAMA), long acting $\beta$ agonist (LABA),
Systematic review and meta-analysis of randomised

\section{WHAT IS ALREADY KNOWN ON THIS TOPIC}

Triple therapy with long acting muscarinic antagonists (LAMA), long acting $\beta 2$ agonists (LABA), and inhaled corticosteroids (ICS) are commonly used in patients with chronic obstructive pulmonary disease (COPD)

Meta-analyses have previously shown that triple therapy using multiple inhalers can improve forced expiratory volume in 1 second (FEV1) and health status, but evidence of triple therapy versus dual therapy for preventing exacerbations is not well documented

\section{WHAT THIS STUDY ADDS}

In a meta-analysis of 21 trials, moderate to high quality evidence indicated that use of triple therapy significantly decreased the risk of moderate or severe COPD exacerbations compared with dual therapy (of ICS and LABA, or LAMA and LABA) or LAMA monotherapy, together with improvements in lung function, and in a range of other clinically relevant measures

Triple therapy should be limited to patients with more severe COPD symptoms that cannot be adequately managed by dual therapy

Results suggested that triple therapy delivered in a single inhaler is non-inferior to the use of multiple inhaler in terms of clinical efficacy, but this needs further examination and inhaled corticosteroid (ICS). Triple therapy was associated with a significantly reduced rate of moderate or severe exacerbations compared with LAMA monotherapy (rate ratio $0.71,95 \%$ confidence interval 0.60 to 0.85$)$, LAMA and LABA $(0.78,0.70$ to $0.88)$, and ICS and LABA $(0.77,0.66$ to 0.91$)$. Trough forced expiratory volume in 1 second (FEV1) and quality of life were favourable with triple therapy. The overall safety profile of triple therapy is reassuring, but pneumonia was significantly higher with triple therapy than with dual therapy of LAMA and LABA (relative risk $1.53,95 \%$ confidence interval 1.25 to 1.87 ).

\section{CONCLUSIONS}

Use of triple therapy resulted in a lower rate of moderate or severe exacerbations of COPD, better lung function, and better health related quality of life than dual therapy or monotherapy in patients with advanced COPD.

STUDY REGISTRATION

Prospero CRD42018077033.

\section{Introduction}

Chronic obstructive pulmonary disease (COPD) represents one of the most important public health challenges because of its high prevalence and related disability and mortality. ${ }^{1}$ Management of COPD mainly relies on inhaled drugs, including inhaled corticosteroids, long acting $\beta 2$ adrenoceptor agonists (LABA), and long acting muscarinic receptor antagonists (LAMA). ${ }^{2}$ Pharmacological management of COPD tends to begin with monotherapy, and step up to dual or triple therapy, as necessary, to control symptoms. $^{3}$

The Global Initiative for Obstructive Lung Disease (GOLD) management strategy recommend triple therapy with inhaled corticosteroids, LABA, and LAMA for GOLD group D patients who develop further exacerbations after receiving initial dual treatment of LABA and LAMA. ${ }^{34}$ Triple therapy using multiple inhalers has been shown to improve forced expiratory volume in one second (FEV1) and health status, ${ }^{5-7}$ but evidence of triple therapy versus dual therapy for preventing exacerbations is not well documented in previous meta-analyses. ${ }^{5-7}$ Recently, several recent large randomised controlled trials have also assessed the efficacy and safety of triple therapy using one fixed dose combination inhaler for patients with COPD at increased exacerbation risk. ${ }^{8-12}$ These studies showed similar results, but no meta-analysis including these trials has been published so far to our knowledge.

Consideration of triple therapy for COPD is common in current clinical practice, ${ }^{1314}$ and the writers of the GOLD guideline stressed the need for research on this 
matter. Therefore, in the present study, we performed a meta-analysis to examine eligible randomised controlled trials assessing the effect of triple inhaled therapy on the risk of exacerbation, and to obtain more comprehensive, accurate, and precise results about this effect.

\section{Methods}

The present study was performed in accordance with the Preferred Reporting Items for Systematic Reviews and Meta-analyses (PRISMA) statement. ${ }^{15}$ This study was prospectively registered in Prospero (https://www. crd.york.ac.uk/PROSPERO/; CRD42018077033).

\section{Search strategy and selection criteria}

An independent review of the PubMed, Embase, Cochrane Library website, Cochrane Central Register of Controlled Trials (CENTRAL), and ClinicalTrials. gov databases was performed from inception to 18 February 2018 (updated on 13 April 2018). The search was conducted with the following keywords: long acting $\beta 2$ agonists (salmeterol, indacaterol, vilanterol, formoterol, olodaterol), long acting antimuscarinics (glycopyrronium, umeclidinium, aclidinium, tiotropium), inhaled corticosteroids (budesonide, fluticasone, beclomethasone, mometasone, ciclesonide), QVA149, Ultibro, Anoro, Duaklir, Spiolto, and chronic obstructive pulmonary disease (eAppendix A). We reviewed reference lists of all primary studies and review articles for additional references. When a duplicate publication of the same trial was found, the study with the most complete, recent, and updated report was included.

Studies that met the following criteria were included in the analysis:

- Patients: stable, moderate to very severe COPD (FEV $1<80 \%$ of predicted value)

- Intervention: triple therapy (of LABA, LAMA, and inhaled corticosteroids)

- Control: dual therapy (of LABA and LAMA, LABA and inhaled corticosteroids, or LAMA and inhaled corticosteroids) or monotherapy (LAMA, LABA, or inhaled corticosteroids)

- Primary outcome: moderate or severe exacerbation

- Other efficacy outcomes: severe exacerbations, death, FEV1, safety (adverse events, serious adverse events, pneumonia and cardiovascular events), and quality of life (St George's respiratory questionnaire [SGRQ] score)

- $\quad$ Study: prospective randomised trials with a duration of at least four weeks.

\section{Data extraction}

Data were independently extracted by two reviewers, and the results were compared to avoid bias from the data extraction process. We extracted the following characteristic information from each study: participants (sample size, mean age, sex, baseline lung function), interventions (intervention treatment and inhaler type, control treatment and inhaler type), outcomes (exacerbations, severe exacerbations, death, adverse events, serious adverse events, pneumonia and cardiovascular events, mean change of FEV1, and SGRQ score), and design (study design, withdrawals, and duration of follow-up). If the data were not reported in the original article, we extrapolated them from the accompanying graphs or ClinicalTrials.gov.

\section{Assessment of risk of bias in included studies}

We assessed the study quality of each included trial according to the recommendations outlined in the Cochrane Handbook for Systematic Reviews of Interventions. We took into account the following items: allocation sequence generation, concealment of allocation, blinding of participants and investigators, incomplete outcome data, selective outcome reporting, and other sources of bias. ${ }^{16}$ The risk of bias was examined by two reviewers concurrently, and discrepancies were resolved by consensus.

\section{Data analysis}

We performed all statistical analyses using the Stata software (version 12.0). We clarified the metric of analysis for primary outcome (moderate or severe exacerbation) as the following: exacerbation rates per patients per year or during follow-up (effect measure: rate ratio), number of patients with at least one exacerbation (effect measure: risk ratio), and time to first exacerbation (effect measure: hazard ratio). Risk ratios and their associated 95\% confidence intervals were used as the effect measures for the outcomes of death and safety, mean differences with corresponding 95\% confidence intervals were used as the effect measures for continuous outcomes (FEV1 and SGRQ score).

Statistical heterogeneity was assessed with the Q test and $\mathrm{I}^{2}$ statistic. $\mathrm{I}^{2}$ values of more than $50 \%$ were considered to represent significant heterogeneity, whereby the random effects model was used; in all other cases, the fixed effects model was used. ${ }^{17}$ We intended to explore potential causes of heterogeneity for effectiveness and safety data on the basis of duration of follow-up ( $<6 v \geq 6$ months) and eosinophil level. We did a sensitivity analysis by excluding trials at high risk of bias. Potential publication bias was evaluated by funnel plots when 10 or more trials were pooled. ${ }^{18}$ All statistical analyses were two sided, and a $P$ value of less than 0.05 was regarded as statistically significant. We also used the GRADE approach (grading of recommendations assessment, development, and evaluation) to rate the quality of evidence and generate absolute estimates of effect for the outcomes. ${ }^{19}$

\section{Patient involvement}

No patients were involved in setting the research question or the outcome measures, nor were they involved in developing plans for design or implementation of the study. No patients were asked to advise on interpretation or writing up of results. There are no plans to disseminate the results of the 
research to study participants or the relevant patient community.

\section{Results}

Eligible studies and characteristics

Of 931 articles obtained from the initial search of the databases, 21 randomised controlled trials (19 publications) were included in the final analysis ${ }^{8-1220-33}$ (fig 1). Excluded studies are summarised in the appendix.

Detailed baseline characteristics of the included randomised controlled trials are summarised in table 1 , and patient inclusion criteria of included trials were summarised in eTable 1 . Six trials used fixed triple therapy (LAMA, LABA, and inhaled corticosteroids contained in one inhaler), and 15 trials used separate triple therapy (three treatments used with different inhalers). Two studies reported twin trials as a pooled result. ${ }^{30} 31$ The duration of the studies ranged from eight to 52 weeks. Two studies directly compared fixed triple therapy with open triple therapy. ${ }^{83}$ Most of the studies were judged to have a low risk of bias according to the Cochrane instrument (fig 2 and fig 3). COPD exacerbation was defined as a sustained worsening of respiratory symptoms that were mild (self managed by the patient), moderate (requiring oral or systemic corticosteroids or antibiotics (or both)), or severe (requiring hospital admission or resulting in death).

\section{Triple therapy versus LAMA}

Ten trials compared triple therapy with LAMA monotherapy in patients with COPD, and the main outcomes and quality of evidence are summarised in

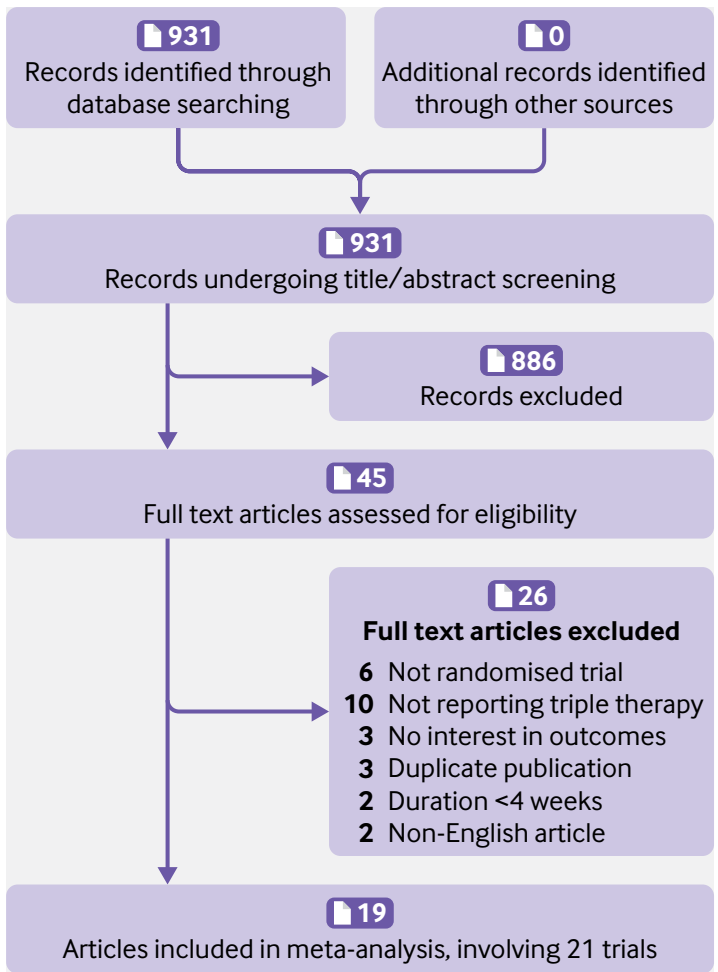

Fig 1 | Flow diagram of trial selection table 2. Compared with LAMA monotherapy, triple therapy significantly reduced the rates of moderate or severe exacerbation (rate ratio $0.71,95 \%$ confidence interval 0.60 to 0.85 ; eFigure $1 \mathrm{~A}$ ), reduced the number of patients with one or more moderate to severe exacerbations (risk ratio $0.74,95 \%$ confidence interval 0.56 to 0.97 ; eFigure $1 \mathrm{~B}$ ), and prolonged time to first moderate or severe exacerbations (hazard ratio 0.69 , $95 \%$ confidence interval 0.54 to 0.88 ; eFigure $1 \mathrm{C}$ ). Triple therapy significantly decreased the rate of severe exacerbation (rate ratio $0.58,0.47$ to 0.72 ; eFigure $2 \mathrm{~A}$ ). We found no statistically significant associations for all cause mortality (eFigure 2B). Use of triple therapy was associated with significant improvements in trough FEV1 (mean difference 0.07, 0.06 to 0.08; eFigure 2C) and mean SGRQ total score $(-2.78,-3.87$ to -1.70 ; eFigure 2D).

Compared with LAMA monotherapy, triple therapy was not associated with increased risk of adverse events, cardiovascular events, and pneumonia events, but was associated with a decreased risk of serious adverse events (eFigures 3-6).

\section{Triple therapy versus LAMA and LABA}

Three trials compared triple therapy with LAMA/ LABA dual therapy in patients with COPD, with the main outcomes and quality of evidence summarised in table 2. Compared with dual therapy of LAMA and LABA, triple therapy significantly reduced the rates of moderate or severe exacerbations (rate ratio $0.78,95 \%$ confidence interval 0.70 to 0.88 ; eFigure $1 \mathrm{~A})$, and prolonged time to first moderate or severe exacerbations (hazard ratio 0.85, 95\% confidence interval 0.79 to 0.91 ; eFigure $1 \mathrm{C}$ ). Triple therapy significantly decreased the rate of severe exacerbation (risk ratio $0.68,95 \%$ confidence interval 0.59 to 0.78 ; eFigure 2A). No statistically significant associations were found for all cause mortality (eFigure 2B). Triple therapy was associated with significant improvements in trough FEV1 (mean difference 0.04, 95\% confidence interval 0.02 to 0.07; eFigure 2C) and mean SGRQ total score $(-1.81,-2.57$ to -1.04 ; eFigure $2 \mathrm{D})$. Compared with dual therapy of LAMA and LABA, triple therapy was not associated with increased risk of adverse events, serious adverse events, or cardiovascular events, but was associated with significantly increased risk of pneumonia events (eFigures 3-6).

\section{Triple therapy versus inhaled corticosteroids and LABA}

Eleven trials compared triple therapy with dual therapy of inhaled corticosteroids and LABA in patients with COPD, with the main outcomes and quality of evidence summarised in table 2. Compared with the dual therapy of inhaled corticosteroids and LABA, triple therapy significantly reduced the rates of moderate or severe exacerbations (rate ratio $0.77,95 \%$ confidence interval 0.66 to 0.91 ; eFigure $1 \mathrm{~A}$ ), reduced the number of patients with at least one moderate or severe exacerbation (risk ratio $0.76,95 \%$ confidence interval, 0.62 to 0.93 ; eFigure $1 \mathrm{~B}$ ), and prolonged time 
Table 1 | Characteristics of studies evaluating triple inhaled therapy

\begin{tabular}{|c|c|c|c|c|c|c|c|c|c|}
\hline \multirow[b]{2}{*}{$\begin{array}{l}\text { Study (first } \\
\text { author, year) }\end{array}$} & \multirow[b]{2}{*}{ Intervention (dose/day) } & \multirow[b]{2}{*}{ Triple therapy } & \multirow[b]{2}{*}{$\begin{array}{l}\text { No of } \\
\text { patients }\end{array}$} & \multirow[b]{2}{*}{$\begin{array}{l}\text { Mean Age } \\
\text { (years) }\end{array}$} & \multirow[b]{2}{*}{ Male (\%) } & & \multirow[b]{2}{*}{$\begin{array}{l}\text { Follow-up } \\
\text { (weeks) }\end{array}$} & \multirow[b]{2}{*}{$\begin{array}{l}\text { Industry } \\
\text { funded }\end{array}$} \\
\hline & & & & & & Trough (L) & $\begin{array}{l}\text { Proportion (\%) of } \\
\text { predicted value }\end{array}$ & & \\
\hline \multicolumn{10}{|c|}{ Triple therapy versus LAMA only } \\
\hline \multirow[t]{2}{*}{ Aaron, $2007^{20}$} & FP $1000 \mu \mathrm{g}$, SAL $100 \mu \mathrm{g}, \mathrm{TIO} 18 \mu \mathrm{g}$ & Separate inhalers & 145 & 67.5 & 57.9 & 1.05 & 39.4 & \multirow[t]{2}{*}{52} & No \\
\hline & $\mathrm{TIO} 18 \mu \mathrm{g}$ & - & 156 & 68.1 & 53.8 & 1.01 & 38.7 & & \\
\hline Cazzola, $2007^{21}$ & FP $1000 \mu \mathrm{g}$, SAL $100 \mu \mathrm{g}, \mathrm{TIO} 18 \mu \mathrm{g}$ & Separate inhalers & 29 & 66.9 & 86.7 & NA & 39.0 & 12 & NA \\
\hline & TIO $18 \mu \mathrm{g}$ & - & 26 & 66.1 & 93.3 & NA & 38.5 & & \\
\hline Hanania, $2012^{22}$ & FP $500 \mu \mathrm{g}, \mathrm{SAL} 100 \mu \mathrm{g}, \mathrm{TIO} 18 \mu \mathrm{g}$ & Separate inhalers & 173 & 61.3 & 50 & 1.70 & NA & 24 & Yes \\
\hline & $\mathrm{TIO} 18 \mu \mathrm{g}$ & - & 169 & 61.0 & 43 & 1.67 & NA & & \\
\hline Hoshino, $2011^{23}$ & FP $500 \mu \mathrm{g}, \mathrm{SAL} 100 \mu \mathrm{g}, \mathrm{TIO} 18 \mu \mathrm{g}$ & Separate inhalers & 16 & 73.0 & 87.5 & 1.36 & 64.6 & 12 & NA \\
\hline & $\mathrm{TIO} 18 \mu \mathrm{g}$ & - & 14 & 73.8 & 100 & 1.28 & 57.1 & & \\
\hline Hoshino, $2013^{24}$ & FP $500 \mu \mathrm{g}, \mathrm{SAL} 100 \mu \mathrm{g}, \mathrm{TIO} 18 \mu \mathrm{g}$ & Separate inhalers & 15 & 73 & 86.7 & 1.38 & NA & 16 & NA \\
\hline & $\mathrm{TIO} 18 \mu \mathrm{g}$ & - & 15 & 73 & 93.3 & 1.29 & NA & & \\
\hline Jung, $2012^{25}$ & FP $500 \mu \mathrm{g}, \mathrm{SAL} 100 \mu \mathrm{g}$, TIO $18 \mu \mathrm{g}$ & Separate inhalers & 223 & 67.0 & 97.3 & 1.22 & 47.4 & 24 & Yes \\
\hline & $\mathrm{TIO} 18 \mu \mathrm{g}$ & - & 232 & 67.8 & 98.7 & 1.21 & 47.5 & & \\
\hline Lee, $2016^{26}$ & BUD $640 \mu \mathrm{g}$, FOR $18 \mu \mathrm{g}, \mathrm{TIO} 18 \mu \mathrm{g}$ & Separate inhalers & 287 & 66.6 & 97.2 & NA & 35.8 & 12 & Yes \\
\hline & $\mathrm{TIO} 18 \mu \mathrm{g}$ & - & 290 & 66.9 & 94.1 & NA & 37.0 & & \\
\hline Maltais, $2013^{27}$ & FP $500 \mu \mathrm{g}, \mathrm{SAL} 100 \mu \mathrm{g}, \mathrm{TIO} 18 \mu \mathrm{g}$ & Separate inhalers & 124 & 62.5 & 56.5 & NA & NA & 8 & Yes \\
\hline & $\mathrm{TIO} 18 \mu \mathrm{g}$ & - & 131 & 62.9 & 53.4 & NA & NA & & \\
\hline Vestbo, $2017^{8}$ & BDP $400 \mu \mathrm{g}$, FOR $24 \mu \mathrm{g}$, GLY $50 \mu \mathrm{g}$ & Fixed inhaler & 1077 & 63.4 & 77 & 1.1 & 36.6 & 52 & Yes \\
\hline & BDP $400 \mu \mathrm{g}$, FOR $24 \mu \mathrm{g}$, TIO $18 \mu \mathrm{g}$ & Separate inhalers & 537 & 62.6 & 74 & 1.1 & 36.7 & & \\
\hline & $\mathrm{TIO} 18 \mu \mathrm{g}$ & - & 1076 & 63.3 & 77 & 1.1 & 36.6 & & \\
\hline Welte, $2009^{28}$ & BDP $640 \mu \mathrm{g}$, FF $18 \mu \mathrm{g}$, TIO $18 \mu \mathrm{g}$ & Separate inhalers & 329 & 62.4 & 76 & 1.1 & 38.1 & 12 & Yes \\
\hline & $\mathrm{TIO} 18 \mu \mathrm{g}$ & - & 331 & 62.5 & 74 & 1.1 & 37.7 & & \\
\hline Triple therapy ve & rsus LAMA and LABA & & & & & & & & \\
\hline Aaron, $2007^{20}$ & FP $1000 \mu \mathrm{g}$, SAL $100 \mu \mathrm{g}, \mathrm{TIO} 18 \mu \mathrm{g}$ & Separate inhalers & 145 & 67.5 & 57.9 & 1.05 & 39.4 & 52 & Yes \\
\hline & SAL $100 \mu \mathrm{g}, \mathrm{TIO} 18 \mu \mathrm{g}$ & - & 148 & 67.6 & 57.4 & 1.00 & 38.0 & & \\
\hline Lipson, $2018^{9}$ & FF $100 \mu \mathrm{g}$, UMEC $625 \mu \mathrm{g}, \mathrm{VI} 25 \mu \mathrm{g}$ & Fixed inhaler & 4151 & 65.3 & 67 & NA & 45.7 & 52 & Yes \\
\hline & UMEC $625 \mu \mathrm{g}, \mathrm{VI} 25 \mu \mathrm{g}$ & - & 2070 & 65.2 & 66 & NA & 45.4 & & \\
\hline Papi, $2018^{10}$ & BDP $348 \mu \mathrm{g}$, FF $20 \mu \mathrm{g}$, GLY $36 \mu \mathrm{g}$ & Fixed inhaler & 764 & 64.4 & 72 & 1.07 & 36.4 & 52 & Yes \\
\hline & IND $85 \mu \mathrm{g}, \mathrm{GLY} 43 \mu \mathrm{g}$ & - & 768 & 64.5 & 72 & 1.07 & 36.4 & & \\
\hline Triple therapy ve & rsus ICS and LABA & & & & & & & & \\
\hline Cazzola, $2007^{21}$ & FP $1000 \mu \mathrm{g}$, SAL $100 \mu \mathrm{g}, \mathrm{TIO} 18 \mu \mathrm{g}$ & Separate inhalers & 29 & 66.9 & 86.7 & NA & 39.0 & 12 & Yes \\
\hline & FP $1000 \mu \mathrm{g}, \mathrm{SAL} 100 \mu \mathrm{g}$ & - & 26 & 64.4 & 86.7 & NA & 36.9 & & \\
\hline Frith, $2015^{29}$ & FP $1000 \mu \mathrm{g}, \mathrm{SAL} 100 \mu \mathrm{g}, \mathrm{GLY} 50 \mu \mathrm{g}$ & Separate inhalers & 257 & 68.2 & 63.4 & 1.52 & 57.36 & 12 & Yes \\
\hline & FP $1000 \mu \mathrm{g}$, SAL $100 \mu \mathrm{g}$, TIO $18 \mu \mathrm{g}$ & Separate inhalers & 258 & 68.0 & 62.0 & 1.49 & 56.86 & & \\
\hline & FP $1000 \mu \mathrm{g}$, SAL $100 \mu \mathrm{g}$ & - & 257 & 67.8 & 67.7 & 1.55 & 57.35 & & \\
\hline Hoshino, $2013^{24}$ & FP $500 \mu \mathrm{g}$, SAL $100 \mu \mathrm{g}, \mathrm{TIO} 18 \mu \mathrm{g}$ & Separate inhalers & 15 & 73 & 86.7 & 1.38 & NA & 16 & Yes \\
\hline & FP $500 \mu \mathrm{g}, \mathrm{SAL} 100 \mu \mathrm{g}$ & - & 16 & 67 & 81.3 & 1.25 & NA & & \\
\hline Lipson, $2017^{11}$ & FF $100 \mu \mathrm{g}$, UMEC $625 \mu \mathrm{g}, \mathrm{VI} 25 \mu \mathrm{g}$ & Fixed inhaler & 911 & 64.2 & 74 & 1.35 & 45.5 & 52 & Yes \\
\hline & BUD $400 \mu \mathrm{g}$, FOR $12 \mu \mathrm{g}$ & - & 899 & 63.7 & 74 & 1.34 & 45.1 & & \\
\hline Lipson $2018^{9}$ & FF $100 \mu \mathrm{g}$, UMEC $625 \mu \mathrm{g}, \mathrm{VI} 25 \mu \mathrm{g}$ & Fixed inhaler & 4151 & 65.3 & 67 & NA & 45.7 & 52 & Yes \\
\hline & $\mathrm{FF} 100 \mu \mathrm{g}, \mathrm{VI} 25 \mu \mathrm{g}$ & - & 4134 & 65.3 & 66 & NA & 45.5 & & \\
\hline Siler, $2015 A^{30}$ & FP $500 \mu \mathrm{g}, \mathrm{SAL} 100 \mu \mathrm{g}$, UMEC $125 \mu \mathrm{g}$ & Separate inhalers & 205 & 63.2 & 69 & 1.35 & 46.7 & 12 & Yes \\
\hline & FP $500 \mu \mathrm{g}, \mathrm{SAL} 100 \mu \mathrm{g}$, UMEC $62.5 \mu \mathrm{g}$ & Separate inhalers & 204 & 62.7 & 65 & 1.31 & 46.8 & & \\
\hline & FP $500 \mu \mathrm{g}, \mathrm{SAL} 100 \mu \mathrm{g}$ & - & 205 & 63.4 & 64 & 1.31 & 47.4 & & \\
\hline Siler, 2015B ${ }^{30}$ & FP $500 \mu \mathrm{g}, \mathrm{SAL} 100 \mu \mathrm{g}$, UMEC $125 \mu \mathrm{g}$ & Separate inhalers & 202 & 65.5 & 59 & 1.21 & 47.6 & 12 & Yes \\
\hline & FP $500 \mu \mathrm{g}, \mathrm{SAL} 100 \mu \mathrm{g}$, UMEC $62.5 \mu \mathrm{g}$ & Separate inhalers & 203 & 64.5 & 69 & 1.16 & 43.9 & & \\
\hline & FP $500 \mu \mathrm{g}$, SAL $100 \mu \mathrm{g}$ & - & 201 & 65.7 & 61 & 1.14 & 44.8 & & \\
\hline Siler, $2015 C^{31}$ & FF $100 \mu \mathrm{g}, \mathrm{VI} 25 \mu \mathrm{g}$, UMEC $125 \mu \mathrm{g}$ & Separate inhalers & 207 & 63.8 & 61 & 1.16 & 45.6 & 12 & Yes \\
\hline & FF $100 \mu \mathrm{g}, \mathrm{VI} 25 \mu \mathrm{g}$, UMEC $62.5 \mu \mathrm{g}$ & Separate inhalers & 206 & 64.9 & 67 & 1.12 & 44.2 & & \\
\hline & FF $100 \mu \mathrm{g}, \mathrm{VI} 25 \mu \mathrm{g}$ & - & 206 & 64.7 & 68 & 1.16 & 45.9 & & \\
\hline Siler, 2015D ${ }^{31}$ & FF $100 \mu \mathrm{g}, \mathrm{VI} 25 \mu \mathrm{g}$, UMEC $125 \mu \mathrm{g}$ & Separate inhalers & 207 & 63.6 & 63 & 1.27 & 47.9 & 12 & Yes \\
\hline & FF $100 \mu \mathrm{g}, \mathrm{VI} 25 \mu \mathrm{g}$, UMEC $62.5 \mu \mathrm{g}$ & Separate inhalers & 206 & 62.6 & 66 & 1.24 & 46.3 & & \\
\hline & FF $100 \mu \mathrm{g}, \mathrm{VI} 25 \mu \mathrm{g}$ & - & 206 & 62.6 & 61 & 1.29 & 47.4 & & \\
\hline Singh, $2016^{12}$ & BDP $400 \mu \mathrm{g}$, FF $24 \mu \mathrm{g}, \mathrm{GLY} 50 \mu \mathrm{g}$ & Fixed inhaler & 687 & 63.3 & 74 & 1.11 & 36.9 & 52 & Yes \\
\hline & $\mathrm{BDP} 400 \mu \mathrm{g}$, FF $24 \mu \mathrm{g}$ & - & 680 & 63.8 & 77 & 1.10 & 36.2 & & \\
\hline Sousa, $2016^{32}$ & ICS, LABA, UMEC $62.5 \mu \mathrm{g}$ & Separate inhalers & 119 & 65.2 & 83 & 1.33 & 47.6 & 12 & Yes \\
\hline & ICS, LABA & - & 117 & 63.1 & 75 & 1.37 & 47.8 & & \\
\hline Fixed triple ther & py versus separate triple therapy & & & & & & & & \\
\hline Bremner, $2018^{33}$ & FF $100 \mu \mathrm{g}$, UMEC $62.5 \mu \mathrm{g}, \mathrm{VI} 25 \mu \mathrm{g}$ & Fixed inhaler & 527 & 66.7 & 74 & 1.25 & 44.5 & 24 & Yes \\
\hline & FF $100 \mu \mathrm{g}$, UMEC $62.5 \mu \mathrm{g}, \mathrm{VI} 25 \mu \mathrm{g}$ & Separate inhalers & 528 & 65.9 & 75 & 1.30 & 45.5 & & \\
\hline Vestbo, $2017^{8}$ & BDP $400 \mu \mathrm{g}$, FOR $24 \mu \mathrm{g}$, GLY $50 \mu \mathrm{g}$ & Fixed inhaler & 1077 & 63.4 & 77 & 1.1 & 36.6 & 52 & Yes \\
\hline & BDP $400 \mu \mathrm{g}$, FOR $24 \mu \mathrm{g}$, TIO $18 \mu \mathrm{g}$ & Separate inhalers & 537 & 62.6 & 74 & 1.1 & 36.7 & & \\
\hline
\end{tabular}

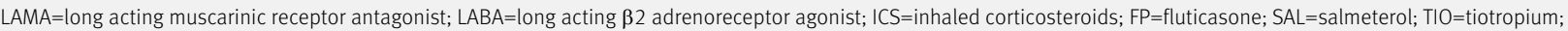

$\mathrm{BDP}=$ beclometasone dipropionate; $\mathrm{FF}=$ formoterol fumarate; $\mathrm{GLY}=$ glycopyrronium; $U M E C=$ umeclidinium; $V I=$ vilanterol; $B U D=$ budesonide; $F O R=$ formoterol; IND=indacaterol; $N A=n o t$ available; FEV1=forced expiratory volume in one second. 


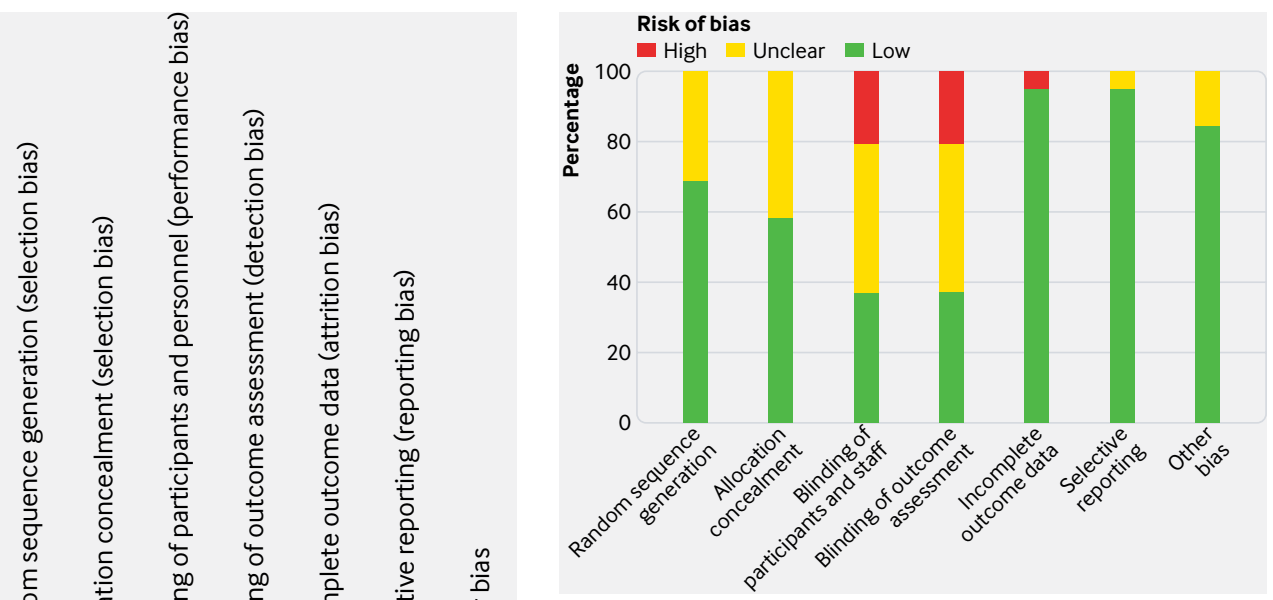

Fig 3 | Risk of bias graph presenting each risk of bias item as percentages across all included studies

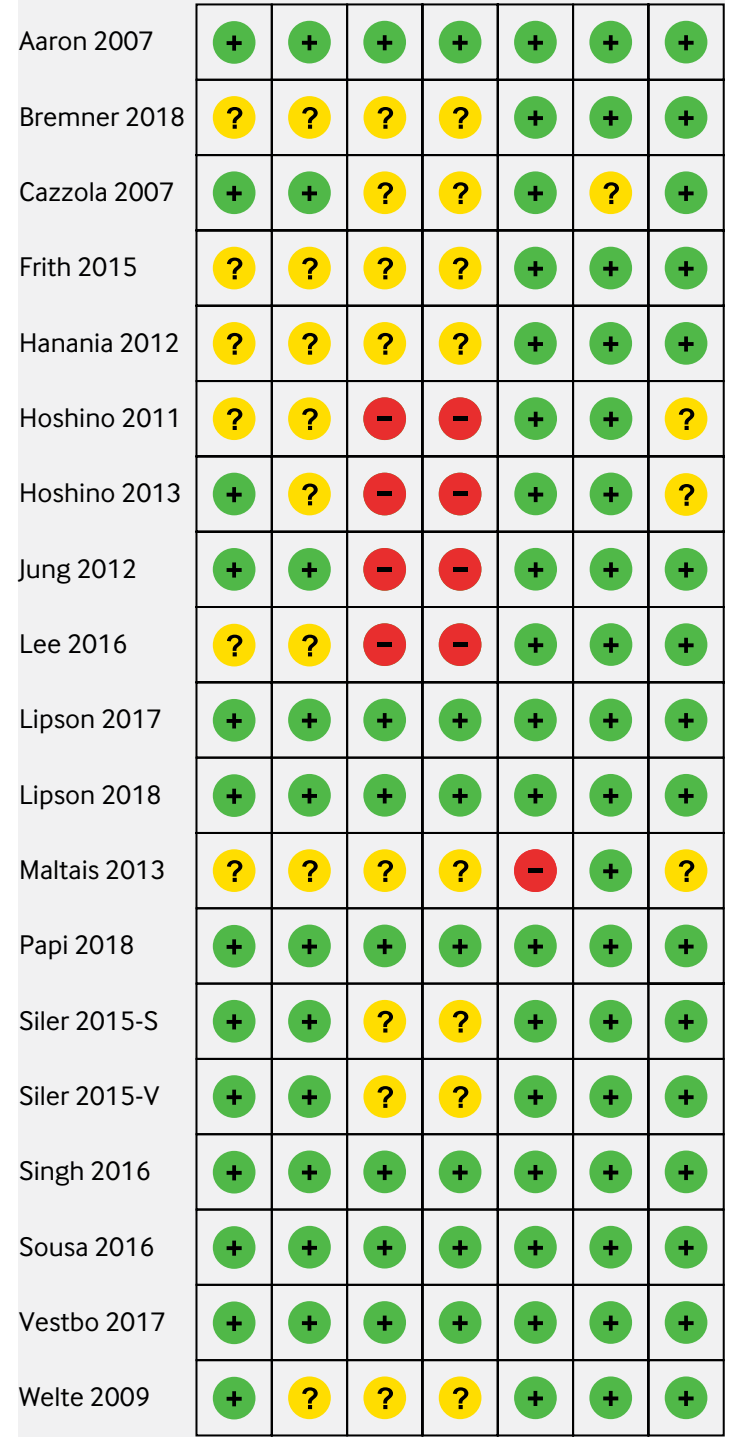

Fig 2 | Risk of bias summary for included studies, showing each risk of bias item for every included study

to the first moderate or severe exacerbation (hazard ratio $0.84,95 \%$ confidence interval 0.79 to 0.90 ; eFigure 1C). Triple therapy significantly decreased the rate of severe exacerbation (risk ratio $0.87,95 \%$ confidence interval 0.75 to 1.00 ; eFigure $2 \mathrm{~A}$ ). No statistically significant associations were found for all cause mortality (eFigure 2B). Triple therapy was associated with significant improvements in trough FEV1 (mean difference $0.11,95 \%$ confidence interval 0.10 to 0.13 ; eFigure 2C) and mean SGRQ total score $(-1.81,-2.28$ to -1.35 ; eFigure 2D). Compared with the dual therapy, triple therapy was not associated with increased risk of adverse events, serious adverse events, cardiovascular events, or pneumonia events (eFigures 3-6).

\section{Fixed triple therapy versus separate triple therapy}

Two trials compared fixed triple therapy with separate triple therapy directly. We found no statistically significant associations for all the outcomes (table 2, eFigures 1-6).

\section{Subgroup and sensitivity analysis}

Subgroup analysis based on duration of follow-up did not suggest any substantial associations with either triple therapy versus dual therapy of inhaled corticosteroids and LABA, or triple therapy versus LAMA only (eTable 3). Descriptive analysis by subgroup of eosinophil counts suggested that blood eosinophil counts were a useful biomarker for predicting which patients were most likely to respond to the triple inhaled therapy (eTable 4). After exclusion of trials with a high risk of bias, the overall findings remained consistent (eTable 5).

\section{Publication bias}

We used funnel plots to access the publication bias, and these results did not show any evidence of obvious bias for the outcome of the number of patients with at least one moderate to severe exacerbation (triple therapy $v$ dual therapy of LABA and inhaled corticosteroids). However, the possibility of obvious publication bias cannot be excluded for the death outcomes (triple therapy $v$ dual therapy of LABA and inhaled corticosteroids; eFigure 7). 


\begin{tabular}{|c|c|c|c|c|c|c|}
\hline Outcomes & No of trials & No/total No of patients & Effect size $(95 \% \mathrm{Cl})^{*}$ & $1^{2}$ & $\mathbf{P}$ & GRADE evidence \\
\hline \multicolumn{7}{|l|}{ Triple therapy versus LAMA (10 trials) } \\
\hline \multicolumn{7}{|l|}{ Moderate to severe exacerbations } \\
\hline Rate of exacerbation & 5 & $2550 / 2020$ & $0.71(0.60$ to 0.85$)$ & 65.4 & 0 & Moderate \\
\hline No of patients with $\geq 1$ moderate to severe exacerbation & 5 & $1159 / 1176$ & $0.74(0.56$ to 0.97$)$ & 50.6 & 0.03 & Moderate \\
\hline Time to first exacerbation & 3 & $2232 / 1695$ & $0.69(0.54$ to 0.88$)$ & 71.6 & 0.002 & Moderate \\
\hline Rate of severe exacerbations & 4 & $1726 / 1730$ & $0.58(0.47$ to 0.72$)$ & 0 & 0 & High \\
\hline All cause mortality & 4 & $2377 / 1851$ & $0.71(0.45$ to 1.10$)$ & 23.3 & 0.13 & Moderate \\
\hline FEV1 trough (L) & 11 & $3483 / 2423$ & 0.07 (0.06 to 0.08$)$ & 0 & 0 & High \\
\hline \multicolumn{7}{|l|}{ Safety } \\
\hline Adverse events & 6 & $2579 / 2046$ & $0.98(0.92$ to 1.04$)$ & 0 & 0.57 & Moderate \\
\hline Serious adverse events & 6 & $2773 / 2252$ & 0.83 (0.71 to 0.98$)$ & 0 & 0.03 & Moderate \\
\hline Cardiovascular events & 2 & $1759 / 1232$ & $0.77(0.45$ to 1.31$)$ & 0 & 0.33 & Low \\
\hline Pneumonia events & 4 & $2269 / 1754$ & 1.30 (0.79 to 2.12$)$ & 0 & 0.30 & Low \\
\hline \multicolumn{7}{|l|}{ Quality of life } \\
\hline SGRQ score & 8 & $3063 / 2056$ & $-2.78(-3.87$ to -1.70$)$ & 0 & 0 & High \\
\hline \multicolumn{7}{|l|}{ Triple therapy versus LAMA and LABA ( 3 trials) } \\
\hline \multicolumn{7}{|l|}{ Moderate to severe exacerbations } \\
\hline Rate of exacerbation & 2 & $4915 / 2838$ & $0.78(0.70$ to 0.88$)$ & 46.3 & 0 & Moderate \\
\hline No of patients with $\geq 1$ moderate to severe exacerbation & 1 & $145 / 148$ & $0.91(0.78$ to 1.07$)$ & NA & NA & Low \\
\hline Time to first exacerbation & 2 & $4915 / 2838$ & $0.85(0.79$ to 0.91$)$ & 0 & 0 & High \\
\hline Rate of severe exacerbations & 3 & $5153 / 3090$ & $0.68(0.59$ to 0.78$)$ & 0 & 0 & High \\
\hline All cause mortality & 3 & $5060 / 2986$ & 0.77 (0.58 to 1.03$)$ & 0 & 0.07 & Moderate \\
\hline FEV1 trough $(\mathrm{L})$ & 3 & $4275 / 2406$ & $0.04(0.02$ to 0.07$)$ & 27.1 & 0 & High \\
\hline \multicolumn{7}{|l|}{ Safety } \\
\hline Adverse events & 3 & $5060 / 2986$ & $1.00(0.93$ to 1.08$)$ & 58.3 & 0.98 & Low \\
\hline Serious adverse events & 3 & $5060 / 2986$ & 0.94 (0.86 to 1.03$)$ & 0 & 0.20 & Moderate \\
\hline Cardiovascular events & 3 & $5060 / 2986$ & $0.97(0.84$ to 1.12$)$ & 27 & 0.70 & Low \\
\hline Pneumonia events & 3 & $5060 / 2986$ & $1.53(1.25$ to 1.87$)$ & 19.7 & 0 & Moderate \\
\hline \multicolumn{7}{|l|}{ Quality of life } \\
\hline SGRQ score & 3 & $4227 / 2386$ & $-1.81(-2.57$ to -1.04$)$ & 0 & 0 & High \\
\hline \multicolumn{7}{|l|}{ Triple therapy versus ICS and LABA (11 trials) } \\
\hline \multicolumn{7}{|l|}{ Moderate to severe exacerbations } \\
\hline Rate of exacerbation & 3 & $5749 / 5713$ & 0.77 (0.66 to 0.91$)$ & 64.0 & 0.003 & Moderate \\
\hline No of patients with $\geq 1$ moderate to severe exacerbation & 8 & $3872 / 3028$ & $0.76(0.62$ to 0.93$)$ & 48.1 & 0.008 & High \\
\hline Time to first exacerbation & 2 & $4838 / 4814$ & $0.84(0.79$ to 0.90$)$ & 0 & 0 & High \\
\hline Rate of severe exacerbations & 1 & $4151 / 4134$ & $0.87(0.75$ to 1.00$)$ & NA & 0.05 & Moderate \\
\hline All cause mortality & 9 & $8023 / 6905$ & 0.88 (0.69 to 1.13$)$ & 0 & 0.33 & Low \\
\hline FEV1 trough $(\mathrm{L})$ & 12 & $6453 / 5348$ & 0.11 (0.10 to 0.13$)$ & 65.1 & 0 & Moderate \\
\hline \multicolumn{7}{|l|}{ Safety } \\
\hline Adverse events & 10 & $8052 / 6931$ & $1.02(0.99$ to 1.04$)$ & 21.5 & 0.78 & Moderate \\
\hline Serious adverse events & 9 & $8023 / 6905$ & 1.01 (0.94 to 1.08$)$ & 42.9 & 0.82 & Moderate \\
\hline Cardiovascular events & 8 & $7904 / 6788$ & 0.99 (0.89 to 1.11) & 0 & 0.92 & Moderate \\
\hline Pneumonia events & 9 & $8023 / 6905$ & $1.11(0.97$ to 1.28$)$ & 2 & 0.13 & Moderate \\
\hline \multicolumn{7}{|l|}{ Quality of life } \\
\hline SGRQ score & 11 & $6383 / 5293$ & $-1.81(-2.28$ to -1.35$)$ & 0 & 0 & High \\
\hline \multicolumn{7}{|l|}{ Fixed triple therapy versus separate triple therapy ( 2 trials) } \\
\hline \multicolumn{7}{|l|}{ Moderate to severe exacerbations } \\
\hline Rate of severe exacerbations & 2 & $1604 / 1065$ & 0.97 (0.85 to 1.11$)$ & 0 & 0.56 & Moderate \\
\hline All cause mortality & 2 & $1604 / 1065$ & $1.18(0.59$ to 2.38$)$ & 0 & 0.64 & Moderate \\
\hline FEV1 trough $(\mathrm{L})$ & 2 & $1604 / 1065$ & $0.01(-0.02$ to 0.04$)$ & 58.6 & 0.52 & Low \\
\hline \multicolumn{7}{|l|}{ Safety } \\
\hline Adverse events & 2 & $1604 / 1065$ & $0.98(0.91$ to 1.05$)$ & 0 & 0.51 & Moderate \\
\hline Serious adverse events & 2 & $1604 / 1065$ & 0.98 (0.79 to 1.22$)$ & 0 & 0.88 & Moderate \\
\hline Cardiovascular events & 2 & $1604 / 1065$ & $1.16(0.75$ to 1.79$)$ & 0 & 0.50 & Low \\
\hline Pneumonia events & 2 & $1604 / 1065$ & $0.88(0.55$ to 1.41$)$ & 24.8 & 0.60 & Low \\
\hline \multicolumn{7}{|l|}{ Quality of life } \\
\hline SGRQ score & 2 & $1553 / 1049$ & $0.20(-1.96$ to 2.35$)$ & 56.7 & 0.93 & Low \\
\hline
\end{tabular}

\section{Discussion}

Despite the current widespread use of triple therapy in patients with COPD who have the highest symptom burden, there are few trials showing a sustained benefit on this treatment combination preventing exacerbations. In this meta-analysis of 21 trials, we found that triple therapy (of LABA, LAMA, and inhaled corticosteroids combined) was associated with a significantly larger reduction in the rate of moderate or severe COPD exacerbations than dual therapy (of 
LAMA and LABA, or inhaled corticosteroids and LABA) or monotherapy (LAMA only). Surrogate outcomes such as spirometry (FEV1) and quality of life (SGRQ score) were favourable, and the overall safety profile of triple therapy is reassuring, but pneumonia was significantly higher with triple therapy than with dual therapy of LAMA and LABA.

\section{Comparison with other studies}

Three meta-analyses have compared the efficacy and safety of tiotropium, LABA, and inhaled corticosteroid treatment compared with that of tiotropium only. ${ }^{5-7}$ All three studies revealed benefits for lung function and quality of life. ${ }^{5-7}$ However, the effect on exacerbation risk is not well documented, which was the focus of the present study. We also compared triple therapy with dual therapy (of LAMA and LABA or of inhaled corticosteroids and LABA), which had not been previously considered. The present review also describes the clinical efficacy of a triple therapy delivered in one inhaler, which had not been previously considered, and our results indicated that single inhalers were not inferior to separate inhalers.

Main findings and interpretation in light of evidence Reductions in exacerbation and mortality risk are the most important outcomes in the management of COPD. ${ }^{1}$ Our results showed that triple therapy significantly reduced the risk of moderate or severe exacerbations compared with LAMA monotherapy and the dual therapies of inhaled corticosteroid and LABA and of LAMA and LABA. All cause mortality was not obviously decreased for triple therapy compared with other treatments. However, most trials did not exceed six months of duration, and thus were limited in the reporting of such final health outcomes. Spirometry is considered a core outcome to measure COPD severity, control, and response to treatment, ${ }^{3}$ and significant improvements in FEV1 were found to be associated with triple therapy. We did not access forced vital capacity because this outcome was seldom reported in the included trials. Moreover, the mean difference in SGRQ score, which is a preferred measurement of COPD control, ${ }^{34}$ showed a consistently positive association with triple therapy. Thus, triple therapy is favourable in the management of COPD in terms of the efficacy outcomes.

Safety results showed a higher incidence of pneumonia in the triple therapy group than in the group receiving dual therapy of LAMA and LABA, with a significant trend to increase pneumonia incidence when compared with LAMA monotherapy (which did not increase incidence significantly). It would be expected, as previous studies have been reported, that treatment including inhaled corticosteroids increases the risk of pneumonia compared with placebo. ${ }^{3536}$ Triple therapy did not increase the risk of cardiovascular adverse events, which was consistent with previous meta-analyses suggesting that dual therapy of LAMA and LABA do not increase the risk of fatal cardiovascular events in patients with COPD compared with LAMA monotherapy or with dual therapy of inhaled corticosteroids and LABA. ${ }^{3738}$ Some of the included trials could have excluded patients at cardiovascular risk. The observed decreased risk of serious adverse events with triple therapy compared with LAMA monotherapy could have been by chance.

Recently, single inhalers containing an inhaled glucocorticoid, LABA, and LAMA have been developed. Two trials compared the fixed triple therapy with separate triple therapy directly. Results from the TRINITY study found that the twice daily, single inhaler use of beclometasone dipropionate, formoterol fumarate, and glycopyrronium bromide combined was not inferior to twice daily use of beclometasone dipropionate, formoterol fumarate, and tiotropium with multiple inhalers. ${ }^{8}$ Similarly, another trial by Bremner and colleagues showed that single inhaler use of $100 \mu \mathrm{g}$ fluticasone furoate, $62.5 \mu \mathrm{g}$ umeclidinium, and $25 \mu \mathrm{g}$ vilanterol was non-inferior to multiple inhaler use of $100 \mu \mathrm{g}$ fluticasone furoate, $25 \mu \mathrm{g}$ vilanterol, and $62.5 \mu \mathrm{g}$ umeclidinium in patients with advanced COPD. ${ }^{33}$

In view of the high level of incorrect inhaler techniques seen in clinical practice, ${ }^{39}$ the availability of a single inhaler product could reduce the likelihood of inhaler use errors. Healthcare resource use data from the FULFIL trial suggest that, in a clinical trial setting over a 52 week timeframe, non-drug costs associated with the management of a single inhaler (containing LAMA, LABA, and inhaled corticosteroids) are lower than twice daily use of LABA and inhaled corticosteroids. ${ }^{40}$ Therefore, a single inhaler regimen of triple therapy offers a simplified dosing option that could improve patient adherence and outcomes, and reduce associated healthcare costs.

Where triple therapy sits in the stepwise approach to managing COPD is not yet known, but triple therapy is widely prescribed in clinical practice. Real life prescription data in the United Kingdom show that $32 \%$ of patients with COPD received triple therapy, of whom $19 \%, 28 \%, 37 \%$, and $46 \%$ were classified as GOLD groups A, B, C, and D, respectively. ${ }^{41}$ The trials in the present meta-analysis included carefully selected patients, of whom many had severe or very severe airflow limitation, were symptomatic at screening despite treatment, and had had at least one documented exacerbation in the past year. In addition, the incidence of COPD exacerbations was low in both the triple therapy groups and control groups of the included trials (eFigure 1), which might imply that both treatments were effective in reducing the rate of COPD exacerbations. Considering that no survival benefit was associated with triple therapy, and increased risk of pneumonia was observed, our results might only apply to patients with symptomatic COPD, severe airflow limitation, and an exacerbation history, and any potential benefit could be lost if triple therapy is expanded to patients with mild COPD.

Therefore, careful identification of patients who might benefit most from triple therapy is required. The TRINITY trial suggested that the effect of triple therapy 
on exacerbation rate was greater in the subgroups with raised eosinophil concentrations. ${ }^{8}$ Similarly, the TRIBUTE trial indicated that triple therapy significantly reduced the exacerbation rate compared with dual therapy in patients with eosinophils of at least $2 \%$, but not in those with eosinophils less than $2 \% .{ }^{10}$ In addition, a post hoc analysis of IMPACT trial showed that the benefits of exacerbation reduction were observed regardless of the patients' blood eosinophil levels at randomisation, with greater reduction in the subset of patients with eosinophil levels of at least 150 cells/ $\mu \mathrm{L} .{ }^{9}$ These findings indicated that blood eosinophil counts might be a useful biomarker for predicting patients most likely to respond to the triple inhaled therapy. Future research could more closely examine the association between eosinophil levels, patient characteristics, exacerbation history, and clinical outcomes of triple therapy.

\section{Limitations}

This study had several limitations. Firstly, patients used dual or triple therapies at baseline, so it is unknown whether the abrupt discontinuation of certain drugs could have contributed to our finding of a lower rate of exacerbations in the triple therapy group than in the monotherapy or dual therapy group. Although analysis was adjusted in some of the included trials, no subgroup analysis was done according to previous treatment. Secondly, because different LAMA, LABA, and inhaled corticosteroids from different devices and in different dosing regimens were used among the included trials, some of the improvements observed could therefore be due to differences in molecules, devices, or dosing regimens. Thirdly, all the included trials were efficacy trials, and there are no effectiveness trials on triple therapy. Future trials are needed to clarify the effectiveness or cost effectiveness of triple therapy in COPD. Finally, we saw a high level of heterogeneity between study results, especially for the primary outcomes, but the directions of effect sizes were consistent among included trials.

\section{Conclusion}

In this meta-analysis of patients with COPD, use of triple therapy resulted in a lower rate of moderate or severe COPD exacerbations and better lung function and health related quality of life than dual therapy (of inhaled corticosteroids and LABA or of LAMA and LABA) or LAMA monotherapy. However, triple therapy did not improve patients' survival, and could increase the risk of pneumonia. Therefore, triple therapy should be limited to patients with more severe COPD symptoms that cannot be adequately managed by dual therapy. Attempts should be made to identify patients with COPD phenotypes (eg, eosinophil levels, patient characteristics, and exacerbation history) most likely to respond to the triple therapy.

Contributors: YZ and IZ contributed equally to this work; YZ, IZ, and $\mathrm{YL}$ contributed to the conception and design of the study; $\mathrm{WL}$ and $\mathrm{CL}$ contributed to the literature search and data extraction; YZ, JZ, and KQ contributed to data analysis and interpretation; $\mathrm{YZ}$ and $\mathrm{JZ}$ conducted the quality assessment; JW and WY contributed to critical revision of the manuscript. All authors contributed to writing the manuscript, and all authors approved the manuscript. JW and WY guarantees the integrity of the work. The lead authors had full access to all the data in the study and the final responsibility for the decision to submit for publication. The corresponding author attests that all listed authors meet authorship criteria and that no others meeting the criteria have been omitted.

\section{Funding: No funding.}

Competing interests: All authors have completed the ICMJE uniform disclosure form at www.icmje.org/coi_disclosure.pdf (available on request from the corresponding author) and declare: no support from any organisation for the submitted work; no financial relationships with any organizations that might have an interest in the submitted work in the previous three years; no other relationships or activities that could appear to have influenced the submitted work.

Ethical approval: Not required.

Data sharing: No additional data available.

The lead author affirms that the manuscript is an honest, accurate, and transparent account of the study being reported; that no important aspects of the study have been omitted; and that any discrepancies from the study as planned have been explained. This is an Open Access article distributed in accordance with the Creative Commons Attribution Non Commercial (CC BY-NC 4.0) license, which permits others to distribute, remix, adapt, build upon this work non-commercially, and license their derivative works on different terms, provided the original work is properly cited and the use is noncommercial. See: http://creativecommons.org/licenses/by-nc/4.0/.

1 GBD 2016 Risk Factors Collaborators. Global, regional, and national comparative risk assessment of 84 behavioural, environmental and occupational, and metabolic risks or clusters of risks, 1990-2016: a systematic analysis for the Global Burden of Disease Study 2016. Lancet 2017;390:1345-422. doi:1016/S0140-6736(17)32366-8

2 Malerba M, Nardin M, Santini G, Mores N, Radaeli A, Montuschi P. Single-inhaler triple therapy utilizing the once-daily combination of fluticasone furoate, umeclidinium and vilanterol in the management of COPD: the current evidence base and future prospects. Ther Adv Respir Dis 2018;12:1753466618760779. doi:10.1177/1753466618760779.

3 Vogelmeier (F, Criner GJ, Martinez FJ, et al. Global strategy for the diagnosis, management, and prevention of chronic obstructive lung disease 2017 report. GOLD executive summary. Am J Respir Crit Care Med 2017;195:557-82. doi:10.1164/rccm.201701-0218PP

4 Global Strategy for the Diagnosis, Management and Prevention of COPD, Global Initiative for Chronic Obstructive Lung Disease (GOLD). 2017. https://goldcopd.org.

5 Rojas-Reyes MX, García Morales OM, Dennis RJ, Karner C. Combination inhaled steroid and long-acting beta -agonist in addition to tiotropium versus tiotropium or combination alone for chronic obstructive pulmonary disease. Cochrane Database Syst Rev 2016;6:CD008532.

6 Liu Y, Shi H, Sun X, et al. Benefits of adding fluticasone propionate/ salmeterol to tiotropium in COPD: a meta-analysis. Eur J Intern Med 2014:25:491-5. doi:10.1016/j.ejim.2014.04.007

7 Kwak MS, Kim E, Jang EJ, Kim HJ, Lee CH. The efficacy and safety of triple inhaled treatment in patients with chronic obstructive pulmonary disease: a systematic review and metaanalysis using Bayesian methods. Int J Chron Obstruct Pulmon Dis 2015;10:2365-76.

8 Vestbo J, Papi A, Corradi M, et al. Single inhaler extrafine triple therapy versus long-acting muscarinic antagonist therapy for chronic obstructive pulmonary disease (TRINITY): a double-blind, parallel group, randomised controlled trial. Lancet 2017;389:1919-29. doi:10.1016/S0140-6736(17)30188-5

9 Lipson DA, Barnhart F, Brealey N, et al, IMPACT Investigators. OnceDaily Single-Inhaler Triple versus Dual Therapy in Patients with COPD. N Engl I Med 2018;378:1671-80. doi:10.1056/NEJMoa1713901

10 Papi A, Vestbo J, Fabbri L, et al. Extrafine inhaled triple therapy versus dual bronchodilator therapy in chronic obstructive pulmonary disease (TRIBUTE): a double-blind, parallel group, randomised controlled trial. Lancet 2018;391:1076-84. doi:10.1016/S01406736(18)30206-X

11 Lipson DA, Barnacle H, Birk R, et al. FULFIL Trial: once-daily triple therapy for patients with chronic obstructive pulmonary disease. Am J Respir Crit Care Med 2017;196:438-46. doi:10.1164/rccm.201703$04490 C$

12 Singh D, Papi A, Corradi M, et al. Single inhaler triple therapy versus inhaled corticosteroid plus long-acting $\beta 2$-agonist therapy for chronic obstructive pulmonary disease (TRILOGY): a double-blind, parallel group, randomised controlled trial. Lancet 2016;388:963-73. doi:10.1016/S0140-6736(16)31354-X 
13 Wurst KE, Punekar YS, Shukla A. Treatment evolution after COPD diagnosis in the UK primary care setting. PLoS One 2014;9:e105296. doi:10.1371/journal.pone.0105296

14 Simeone JC, Luthra R, Kaila S, et al. Initiation of triple therapy maintenance treatment among patients with COPD in the US. Int J Chron Obstruct Pulmon Dis 2016;12:73-83. doi:10.2147/COPD. S122013

15 Liberati A, Altman DG, Tetzlaff J, et al. The PRISMA statement for reporting systematic reviews and meta-analyses of studies that evaluate healthcare interventions: explanation and elaboration. $B M$ J 2009;339:b2700. doi:10.1136/bmj.b2700

16 Higgins JP, Altman DG, Gøtzsche PC, et al, Cochrane Bias Methods Group; Cochrane Statistical Methods Group. The Cochrane Collaboration's tool for assessing risk of bias in randomised trials. BMJ 2011;343:d5928.

17 Higgins JPT, Green S, eds. Cochrane Collaboration. Cochrane Handbook for Systematic Reviews of Interventions. Wiley-Blackwell, 2008. doi:10.1002/9780470712184

18 Sobieraj DM, Baker WL, Nguyen E, et al. Association of inhaled corticosteroids and long-acting muscarinic antagonists with asthma control in patients with uncontrolled, persistent asthma: a systematic review and meta-analysis. JAMA 2018;319:1473-84. doi:10.1001/ jama.2018.2757

19 Guyatt G, Oxman AD, Akl EA, et al. GRADE guidelines: 1. IntroductionGRADE evidence profiles and summary of findings tables. J Clin Epidemiol 2011;64:383-94. doi:10.1016/j.jclinepi.2010.04.026

20 Aaron SD, Vandemheen KL, Fergusson D, et al, Canadian Thoracic Society/Canadian Respiratory Clinical Research Consortium. Tiotropium in combination with placebo, salmeterol, or fluticasonesalmeterol for treatment of chronic obstructive pulmonary disease: a randomized trial. Ann Intern Med 2007;146:545-55 doi:10.7326/0003-4819-146-8-200704170-00152

21 Cazzola M, Andò F, Santus P, et al. A pilot study to assess the effects of combining fluticasone propionate/salmeterol and tiotropium on the airflow obstruction of patients with severe-to-very severe COPD. Pulm Pharmacol Ther 2007;20:556-61. doi:10.1016/j. pupt.2006.06.001

22 Hanania NA, Crater GD, Morris AN, Emmett AH, O’Dell DM, Niewoehner DE. Benefits of adding fluticasone propionate/salmeterol to tiotropium in moderate to severe COPD. Respir Med 2012;106:91101. doi:10.1016/j.rmed.2011.09.002

23 Hoshino M, Ohtawa J. Effects of adding salmeterol/fluticasone propionate to tiotropium on airway dimensions in patients with chronic obstructive pulmonary disease. Respirology 2011;16:95101. doi:10.1111/j.1440-1843.2010.01869.x

24 Hoshino M, Ohtawa J. Effects of tiotropium and salmeterol/ fluticasone propionate on airway wall thickness in chronic obstructive pulmonary disease. Respiration 2013;86:280-7. doi:10.1159/000351116

25 Jung KS, Park HY, Park SY, et al, Korean Academy of Tuberculosis and Respiratory Diseases study group, Korea Chronic Obstructive Pulmonary Disease study group. Comparison of tiotropium plus fluticasone propionate/salmeterol with tiotropium in COPD: a randomized controlled study. Respir Med 2012;106:382-9. doi:10.1016/j.rmed.2011.09.004

26 Lee SD, Xie CM, Yunus F, et al. Efficacy and tolerability of budesonide/ formoterol added to tiotropium compared with tiotropium alone in patients with severe or very severe COPD: A randomized, multicentre study in East Asia. Respirology 2016;21:119-27. doi:10.1111/ resp. 12646

27 Maltais F, Mahler DA, Pepin V, et al. Effect of fluticasone propionate/salmeterol plus tiotropium versus tiotropium on walking endurance in COPD. Eur Respir / 2013;42:539-41. doi:10.1183/09031936.00074113
28 Welte T, Miravitlles M, Hernandez P, et al. Efficacy and tolerability of budesonide/formoterol added to tiotropium in patients with chronic obstructive pulmonary disease. Am J Respir Crit Care Med 2009;180:741-50. doi:10.1164/rccm.200904-04920C

29 Frith PA, Thompson PJ, Ratnavadivel R, et al, Glisten Study Group. Glycopyrronium once-daily significantly improves lung function and health status when combined with salmeterol/fluticasone in patients with COPD: the GLISTEN study, a randomised controlled trial. Thorax 2015;70:519-27. doi:10.1136/thoraxinl-2014-206670

30 Siler TM, Kerwin E, Singletary K, Brooks J, Church A. Efficacy and safety of umeclidinium added to fluticasone propionate/salmeterol in patients with COPD: results of two randomized, double-blind studies. COPD 2016:13:1-10. doi:10.3109/15412555.2015.1034256

31 Siler TM, Kerwin E, Sousa AR, Donald A, Ali R, Church A. Efficacy and safety of umeclidinium added to fluticasone furoate/vilanterol in chronic obstructive pulmonary disease: Results of two randomized studies [correction in: Respir Med 2015;109:1493]. Respir Med 2015;109:1155-63. doi:10.1016/j.rmed.2015.06.006

32 Sousa AR, Riley JH, Church A, Zhu CQ, Punekar YS, Fahy WA. The effect of umeclidinium added to inhaled corticosteroid/long-acting $\beta 2$-agonist in patients with symptomatic COPD: a randomised, double-blind, parallel-group study. NPJ Prim Care Respir Med 2016;26:16031. doi:10.1038/npjpcrm.2016.31

33 Bremner PR, Birk R, Brealey N, Ismaila AS, Zhu CQ, Lipson DA. Single-inhaler fluticasone furoate/umeclidinium/vilanterol versus fluticasone furoate/vilanterol plus umeclidinium using two inhalers for chronic obstructive pulmonary disease: a randomized noninferiority study. Respir Res 2018;19:19. doi:10.1186/s12931-0180724-0

34 Meguro M, Barley EA, Spencer S, Jones PW. Development and validation of an improved, COPD-specific version of the St. George respiratory questionnaire. Chest 2007;132:456-63. doi:10.1378/ chest.06-0702

35 Kew KM, Seniukovich A. Inhaled steroids and risk of pneumonia for chronic obstructive pulmonary disease. Cochrane Database Syst Rev 2014:(3):CD010115

36 Horita N, Goto A, Shibata Y, et al. Long-acting muscarinic antagonist (LAMA) plus long-acting beta-agonist (LABA) versus LABA plus inhaled corticosteroid (ICS) for stable chronic obstructive pulmonary disease (COPD). Cochrane Database Syst Rev 2017;2:CD012066.

37 Xia N, Wang H, Nie X. Inhaled long-acting $\beta 2$-agonists do not increase fatal cardiovascular adverse events in COPD: a meta-analysis. PLoS One 2015:10:e0137904. doi:10.1371/journal.pone.0137904

38 Rodrigo GJ, Castro-Rodriguez JA, Nannini LJ, Plaza Moral V, Schiavi EA. Tiotropium and risk for fatal and nonfatal cardiovascular events in patients with chronic obstructive pulmonary disease: systematic review with meta-analysis. Respir Med 2009;103:1421-9. doi:10.1016/j.rmed.2009.05.020

39 Melani AS, Bonavia M, Cilenti V, et al, Gruppo Educazionale Associazione Italiana Pneumologi Ospedalieri. Inhaler mishandling remains common in real life and is associated with reduced disease control [correction in: Respir Med 2012;106:757]. Respir Med 2011:105:930-8 doi:10.1016/j.rmed 2011.01.005

40 Ismaila AS, Birk R, Shah D, et al. Once-daily triple therapy in patients with advanced COPD: healthcare resource utilization data and associated costs from the FULFIL Trial. Adv Ther 2017;34:2163-72. doi:10.1007/s12325-017-0604-x

41 Brusselle G, Price D, Gruffydd-Jones K, et al. The inevitable drift to triple therapy in COPD: an analysis of prescribing pathways in the UK. Int / Chron Obstruct Pulmon Dis 2015;10:2207-17.

\section{Web appendix: Supplementary appendix}

